\title{
Análisis de las lesiones encefálicas en resonancia magnética en pacientes con señales transitorias de alta intensidad detectadas por Doppler transcraneal
}

\section{Analysis of Encephalic Lesions in Magnetic Resonance Imaging in patients with High Intensity Transient Signals detected on Transcranial Doppler}

\author{
Alberto Surur $^{1}$ Lucas M. Pessini Ferreira ${ }^{1}$ Jorge A. Galíndez ${ }^{1}$ Juan J. Martín ${ }^{2}$ \\ ${ }^{1}$ Servicio de Diagnóstico por Imágenes, Sanatorio Allende, Córdoba, \\ Argentina \\ 2 Servicio de Neurología, Sanatorio Allende, Córdoba, Argentina \\ Address for correspondence Lucas M. Pessini Ferreira, Obispo Oro 42, \\ 1er subsuelo, CP 5000, Córdoba, Argentina \\ (e-mail: lucaspssn@gmail.com).
}

Rev Argent Radiol 2019;83:141-150.

\section{Resumen \\ Palabras clave \\ - ultrasonografía Doppler transcraneal \\ - imagen por resonancia magnética \\ - foramen oval permeable}

\section{Abstract}

Objetivo Describir los hallazgos en resonancia magnética (RM) de encéfalo en pacientes menores de 65 años que fueron estudiados por Doppler transcraneal (DTC) con contraste de microburbujas, con antecedentes de accidente cerebrovascular (ACV) criptogénico y sospecha de foramen oval permeable (FOP).

Materiales y métodos Este estudio transversal retrospectivo incluyó pacientes de ambos sexos, menores de 65 años.

Resultados Nuestra muestra ( $n=47,47 \%$ masculino y $53 \%$ femenino, edad media de 42 años) presentó señales transitorias de alta intensidad (HITS, por su sigla en inglés) positivo en el $61,7 \%$ y HITS-negativo en el 38,3\%. En pacientes HITS-positivo, predominaron las lesiones a nivel de las fibras en $\mathrm{U}$ subcorticales, únicas o múltiples con distribución bilateralmente simétrica. En pacientes con HITS moderados, predominaron las lesiones en el territorio vascular de la circulación posterior.

Conclusión En pacientes menores de 65 años con ACV criptogénico y lesiones en fibras en $U$ subcorticales, únicas o múltiples con distribución bilateral y simétrica, debe tenerse en cuenta un FOP como posible causa de dichas lesiones.

Objectives To analyze the findings on brain magnetic resonance imaging (MRI) in patients less than 65 years of age with history of cryptogenic stroke and suspected patent foramen ovale (PFO) who were studied with Contrast-Transcranial Doppler. Materials and Methods This transversal retrospective study included both, men and women less than 65 years of age.

\section{received}

December 6, 2017

accepted

July 30, 2019
DOI https://doi.org/

10.1055/s-0039-1696696.

ISSN 1852-9992.
Copyright @ $\odot$ 2019, Sociedad Argentina de Radiología. Publicado por Thieme Revinter Publicações Ltda., Rio de Janeiro, Brazil. Todos los derechos reservados.
License terms

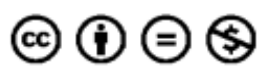




\section{Keywords}

- transcranial Doppler ultrasonography

- magnetic resonance imaging

- patent foramen ovale
Results Our sample ( $n=47,47 \%$ male and $53 \%$ female, average age 42 years old) had High Intensity Transient Signals (HITS)-positive in 61.7\% and HITS-negative in 38.3\%. In HITS-positive patients, lesions were predominantly located on the subcortical U fibers, lone or multiple bilateral symmetric distributions. In patients with moderate-severity HITS, the posterior circulation was the most affected.

Conclusion In patients less than 65 years of age with cryptogenic stroke with lesions affecting the subcortical $U$ fibers, with unique or multiple bilateral symmetric distributions, a PFO should be considered as an underlying cause.

\section{Introducción}

Según datos de la American Heart Association (AHA), la prevalencia de accidente cerebrovascular (ACV) en pacientes de entre 20 y 59 años es de hasta el $2,4 \%$ de la población (mayor prevalencia en mujeres de entre 40 y 59 años ). ${ }^{1,2}$ En Argentina, en un estudio realizado en la ciudad de Junín, se detectó una prevalencia de 868,1 casos por 100.000 habitantes (tasa ajustada a la población mundial: 473,4 por 100.000 habitantes), con un $79,6 \%$ de eventos isquémicos y un $20,4 \%$ hemorrágicos. ${ }^{3}$ Se han reportado múltiples factores de riesgo (FR) predisponentes para el desarrollo de $\mathrm{ACV}$ isquémico. ${ }^{4,5} \mathrm{En}$ cuanto a su etiología, se reconocen cinco categorías de acuerdo a los criterios del Trial of ORG 10172 in Acute Stroke Treatment (TOAST: aterosclerosis, cardioembólico, enfermedad de pequeño vaso, de otra etiología determinada, de etiología indeterminada). ${ }^{6}$ En pacientes jóvenes con ACV isquémicos, los de tipo criptogénico ("de etiología indeterminada" en su descripción original) alcanzan hasta un 30\% y $40 \%$ en la mayoría de los registros y bases de datos. ${ }^{7-13}$

Múltiples estudios muestran una asociación entre foramen oval permeable (FOP) y ACV criptogénico en pacientes de todas las edades, sobre todo en menores de 65 años. ${ }^{14-20}$ Sin embargo, existe poca evidencia acerca de cuáles son los hallazgos en resonancia magnética (RM) de encéfalo que se asocian con mayor frecuencia a ACV a partir de embolias paradójicas con señales transitorias de alta intensidad (HITS, por su sigla en inglés) positivos.

Las modalidades de imagen propuestas incluyen: 1) Resonancia magnética (RM) de encéfalo, 2) Doppler transcraneal (DTC) con contraste de microburbujas, para detectar HITS, que se generan a partir de embolias paradójicas extracraneales. $^{21,22}$

\section{Objetivo}

Describir los hallazgos en RM de encéfalo en pacientes menores de 65 años que fueron estudiados por DTC con contraste de microburbujas, con antecedentes de ACV y sospecha de FOP.

\section{Materiales y métodos}

En el presente estudio retrospectivo se incluyeron pacientes de ambos sexos, menores de 65 años, que fueron estudiados mediante RM y DTC con contraste de microburbujas por sospecha clínica de embolia paradójica, con antecedentes de $\mathrm{ACV}$ y/o de FOP, teniendo en cuenta sus factores de riesgo (FR).

\section{Protocolos de estudio}

FR: Obtenidos por un médico neurólogo y agregados a la historia clínica de cada paciente, siguiendo los criterios establecidos por la AHA. ${ }^{2}$

DTC: Realizado en una única ocasión, siendo cada estudio efectuado íntegramente por un médico radiólogo con experiencia en la técnica, con la asistencia y control clínico a cargo de un médico neurólogo, también con experiencia en la misma. Se utilizó un ecógrafo Philips ${ }^{\circledR}$ HD11XE con transductor sectorial S4-2 (rango de frecuencia de 4 a $2 \mathrm{MHz}$ ) de alta resolución, siguiendo la técnica de Teague y Sharma. ${ }^{23}$ Previo al procedimiento, se evaluó que el paciente realizara adecuadamente la maniobra de Valsalva, lo cual se puso de manifiesto como una disminución en las velocidades de flujo y de la frecuencia cardíaca en el espectro de onda del flujo arterial intracraneal. Posteriormente, se formó una suspensión con microburbujas (-Fig. 1) mezclando $0,2 \mathrm{ml}$ de aire, $0,5 \mathrm{ml}$ de sangre del paciente y $5 \mathrm{ml}$ de solución fisiológica en dos jeringas de $10 \mathrm{ml}$ conectadas por una llave de tres vías, y esa última a la vena antecubital del paciente. Esa suspensión fue inmediatamente inyectada en bolo y conjuntamente con maniobras de Valsalva para la detección de HITS mediante DTC a nivel de la circulación intracraneal, explorando por vía temporal las arterias cerebrales media, bifurcación carotídea, cerebral anterior y cerebrales posteriores; y por ventana occipital las arterias vertebrales (segmento V4) y la arteria basilar (-Fig. 2). En casos dudosos se repitió el mismo procedimiento. Se obtuvo el espectro de ondas Doppler de manera continua en el momento de inyección del medio de contraste.

RM: Se utilizaron Resonadores Magnéticos Philips ${ }^{\circledR}$ Intera 1.5-T y Siemens ${ }^{\circledR}$ Magnetom Essenza 1.5-T con bobina Sense de cabeza. Se obtuvieron imágenes utilizando secuencias Fast Spin Eco (FSE) T2y Fluid-attenuated inversion recovery (FLAIR), Gradiente de Eco (GRE) T2, Difusión (DWI) y coeficiente de difusión aparente (Apparent Diffusion Coefficient-ADC) y Spin Eco(SE) ponderada en T1 con cortes de $5 \mathrm{~mm}$ de espesor. Se incluyó examen por Angio-RM 3D Time of Flight (TOF) de vasos intracraneales con reconstrucciones Maximum Intensity Projection (MIP).

\section{Definición de criterios}

FR: Como FR tradicionales, consideramos: hipertensión arterial, diabetes, tabaquismo, dislipemia, arritmias tipo fibrilación auricular y cardiopatía isquémica. Como FR no tradicionales: hiperhomocisteinemia, uso de anticonceptivos orales y síndrome antifosfolipídico. 


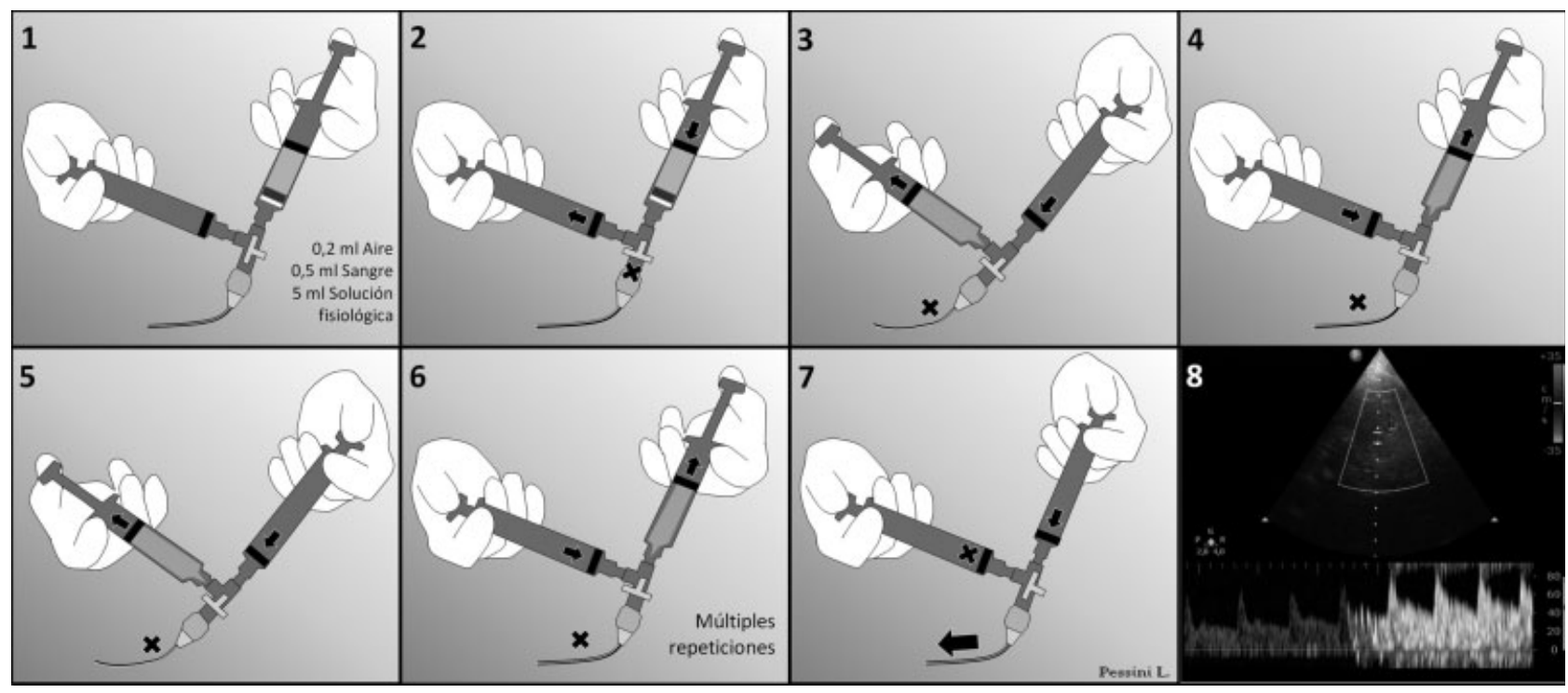

Fig. 1 Esquema representativo de los pasos a seguir para formar la suspensión de microburbujas: 1 a 8 en orden cronológico. Paso 1: Aspiración de sangre por una vía periférica, hacia la jeringa con Aire y Solución Fisiológica. Pasos 2 al 6: Se realiza el mezclado de las diferentes soluciones con producción de microburbujas. Paso 7: Inyección por vía periférica. Paso 8: Registro positivo de HITS en el espectro.

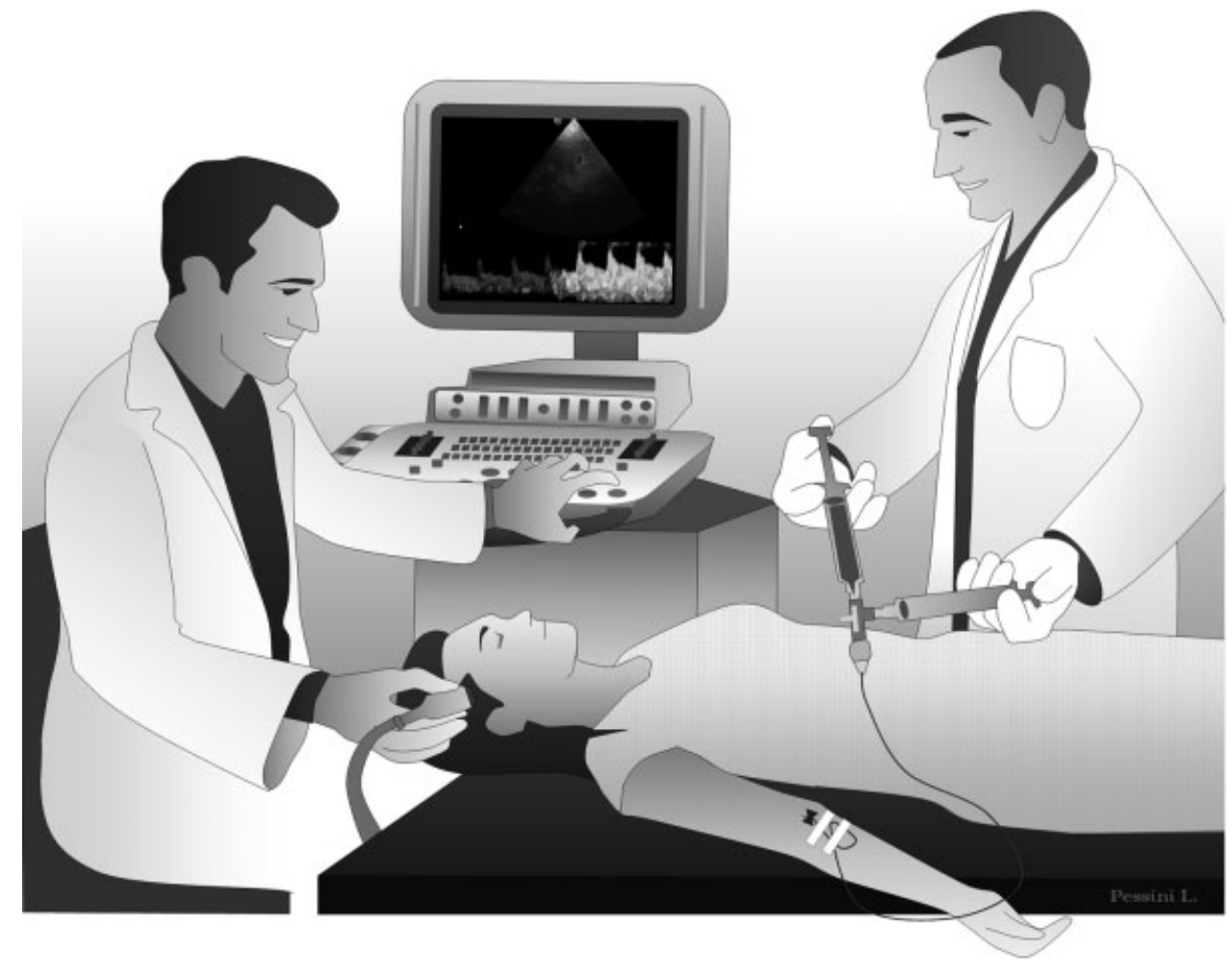

Fig. 2 Esquema representativo de la disposición del paciente y de los médicos (radiólogo y neurólogo) al momento de efectuar el examen.

DTC: Para ser consideradas como HITS, las señales detectadas debieron cumplir con los siguientes criterios: ser transitorias (menos de 300 milisegundos de duración), de alta intensidad (tres decibeles por encima de la circulación de fondo), tener señal unidireccional en el espectro de velocidad de flujo y coincidir con un sonido característico de chasquido. ${ }^{24}$ Para definir su grado, se utilizaron los criterios de Blersch, ${ }^{22}$ reconociendo cuatro posibles categorías: (1) C0: $\mathrm{Sin}$ HITS. (2) C1: menos de 10 HITS. (3) C2: entre 10 y 25 HITS sin cortina. (4) C3: HITS en cortina (-Fig. 3).
RM: Se definieron como lesiones a aquellos focos hiperintensos con respecto a la señal de la sustancia blanca en secuencias FLAIR, y las mismas fueron estudiadas teniendo en cuenta ( - Tabla 1 ) su localización, distribución (número y simetría de lesiones) y el territorio vascular afectado.

En cuanto a la localización de lesiones, se consideraron en el nivel supratentorial (ST) las lesiones de sustancia gris cortical (SG), núcleos de la base (NB), fibras en $U$ subcorticales (SC), nivel periventricular (PV), corona 

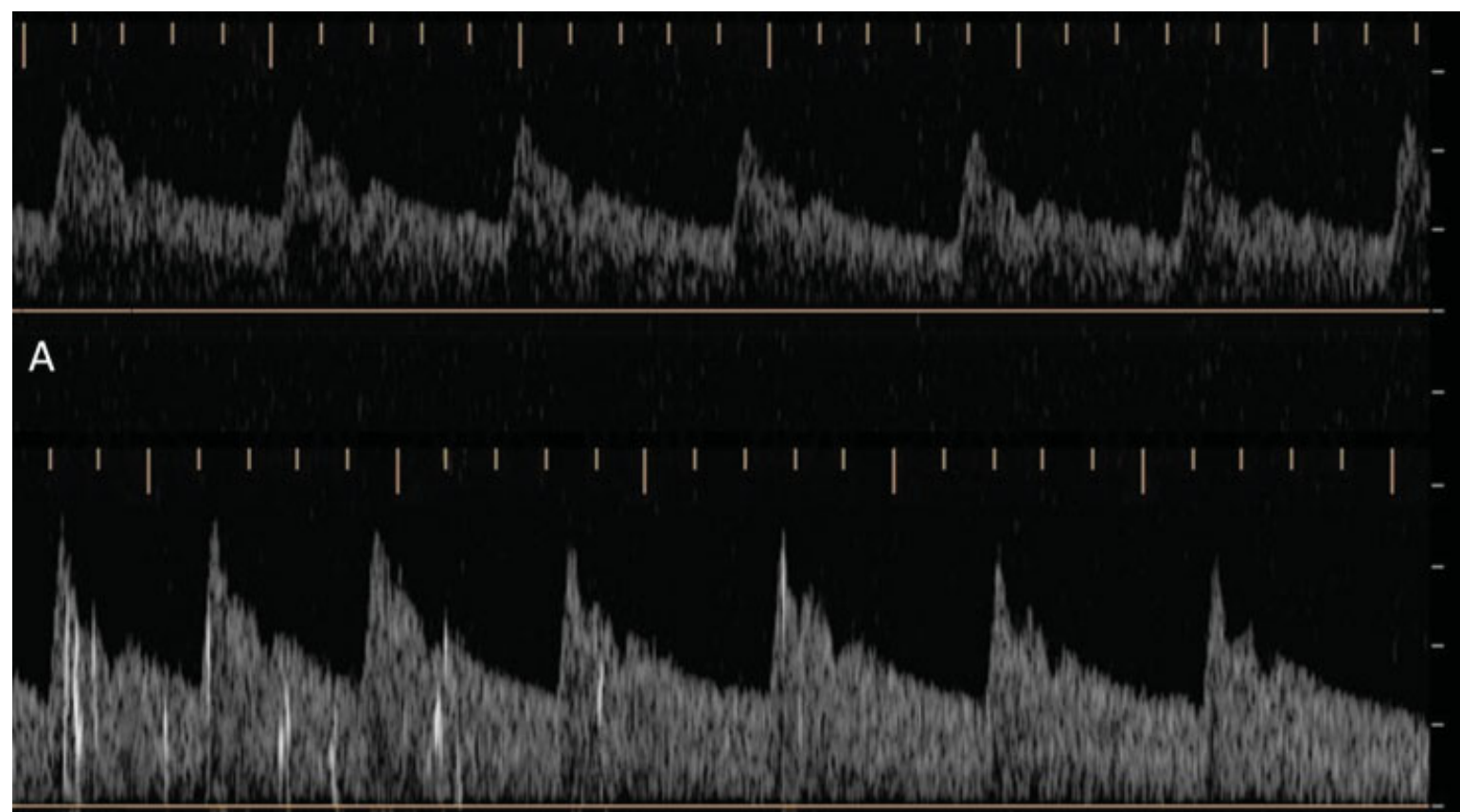

B
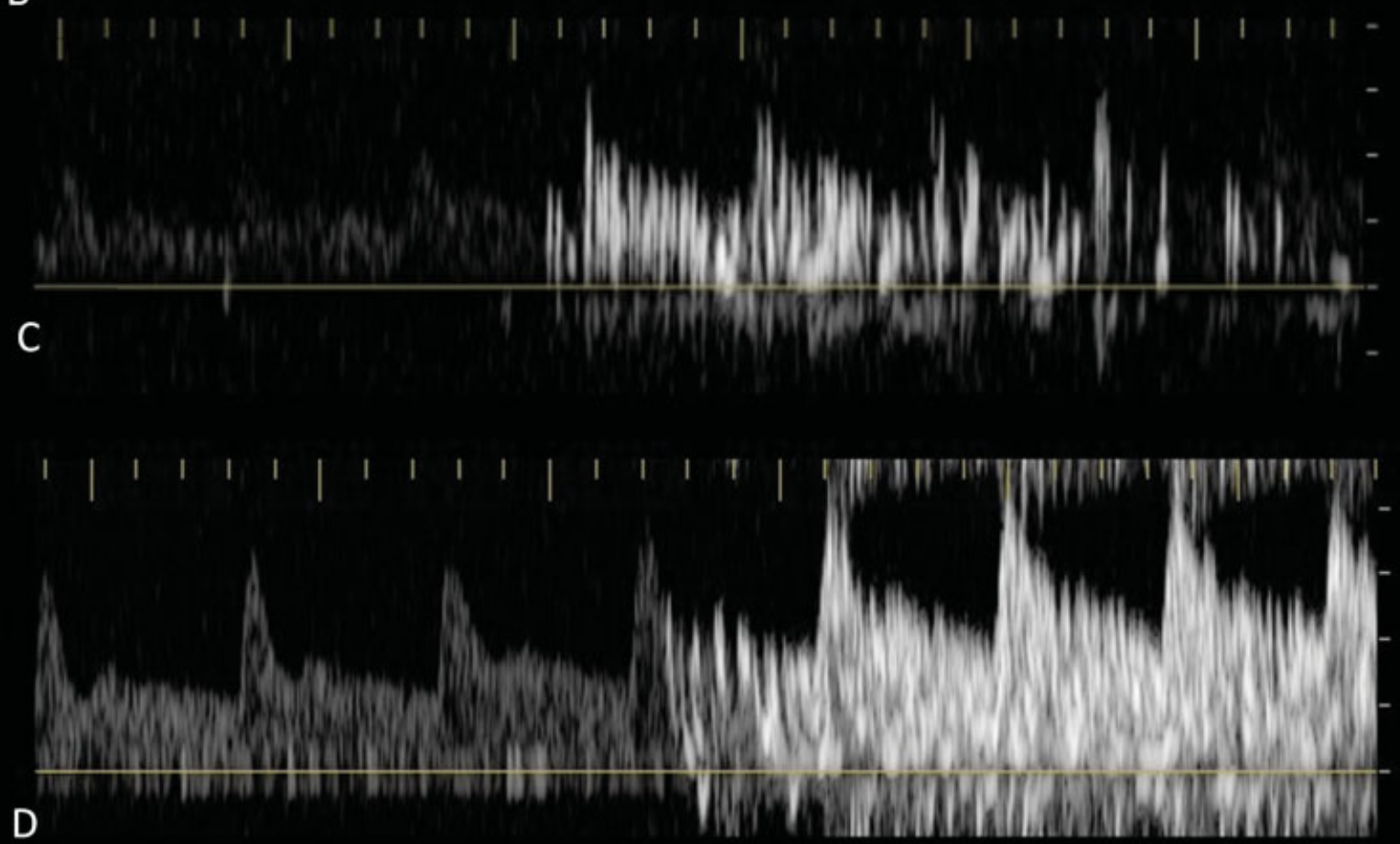

Fig. 3 Clases de HITS detectadas por Doppler transcraneal: A: C0, Sin HITS. B: C1, menos de 10 HITS. C: C2, entre 10 y 25 HITS sin cortina. D: C3, HITS en cortina.

radiada y centro semioval (CR) y cuerpo calloso (CC), y en el nivel infratentorial (IT) las lesiones en región del tronco encefálico (RT) y en región del cerebelo (RC).

En cuanto a la distribución, se consideraron como categorías los pacientes sin lesiones (SL), con lesión única (LU), con lesiones múltiples unilaterales (MU) y con lesiones múltiples bilaterales (MB).

En cuanto a territorios vasculares, se estudió en la circulación anterior (CA): la arteria cerebral anterior (ACA) y la arteria cerebral media (ACM). En la circulación posterior $(\mathrm{CP})$ : las arterias del tronco encefálico (AT) y las arterias del cerebelo (AC).

\section{Análisis estadístico}

Para el análisis estadístico se empleó el test exacto de Fisher con cálculo de $\mathrm{p}$ bilateral, considerando estadísticamente significativos los valores menores a 0,05 . Para definir si el tipo de lesiones guarda relación o no con FR 
Tabla 1 Abreviaturas utilizadas en el presente trabajo para localización, distribución y territorio vascular de lesiones

\begin{tabular}{|l|}
\hline Localización \\
\hline Supratentorial (ST) \\
\hline Sustancia gris (SC) \\
\hline Núcleos de la base (NB) \\
\hline Fibras en U subcorticales (SC) \\
\hline Periventricular (PV) \\
\hline Corona radiada (CR) \\
\hline Cuerpo calloso (CC) \\
\hline Infratentorial (IT) \\
\hline Región del tronco (RT) \\
\hline Región del cerebelo (RC) \\
\hline Distribución \\
\hline Sin lesiones (SL) \\
\hline Lesión única (LU) \\
\hline Múltiples unilaterales (MU) \\
\hline Múltiples bilaterales (MB) \\
\hline Territorio vascular \\
\hline Circulación anterior (CA) \\
\hline Arteria cerebral anterior $(A C A)$ \\
\hline Arteria cerebral media $(A C M)$ \\
\hline Circulación posterior (CP) \\
\hline Arterias del tronco (AT) \\
\hline Arterias cerebelosas (AC) \\
\hline Ambas, CA y CP (A) \\
\hline
\end{tabular}

preexistentes (por ejemplo, hipertensión arterial o diabetes), se utilizó también el test exacto de Fisher con cálculo de p bilateral.

\section{Aspectos éticos}

El presente estudio cuenta con la aprobación del Comité de Ética de la Institución en la que ha sido realizado. Se ha mantenido el anonimato de todos los pacientes incluidos. Los autores declaran no tener conflictos de interés relacionados con el presente estudio.

\section{Resultados}

Se obtuvo una muestra (n) de 47 pacientes menores de 65 años (media: 42, rango: 17-54, DS $\pm 12,4$ ), los cuales fueron estudiados con RM y DTC con inyección de microburbujas, por sospecha de ACV (47\% de sexo masculino y 53\% de sexo femenino). De los pacientes estudiados (-Tablas 2, - Fig. 4), el $61,7 \%$ fue HITS-positivo en el DTC (27,6\% fueron C1, 21,3\% C2 y $12,8 \%$ C3), y el 38,3\% HITS-negativo (C0).

\section{Localización lesional}

Teniendo en cuenta que es posible la coexistencia de lesiones en más de una localización, observamos que (-Tabla 2 y -Fig. 5):
Tabla 2 Localización, distribución y territorio vascular afectado por las lesiones, tanto en pacientes HITS-positivo, como en HITS-negativo

\begin{tabular}{|c|c|c|c|c|}
\hline & Lesiones & HITS (\%) & & Valor $\mathrm{p}$ \\
\hline Localización & totales (\%) & POSITIVO & NEGATIVO & \\
\hline$S T$ & 78,7 & 63,2 & 36,8 & 0,7155 \\
\hline SG & 19,1 & 61,5 & 38,5 & \\
\hline$N B$ & 14,9 & 60,0 & 40,0 & \\
\hline SC & 72,3 & 83,3 & 16,7 & 0,0294 \\
\hline$P V$ & 19,1 & 64,3 & 35,7 & \\
\hline$C R$ & 31,9 & 47,8 & 52,2 & 0,0753 \\
\hline CC & 2,1 & 50,0 & 50,0 & \\
\hline IT & 19,1 & 63,6 & 36,4 & \\
\hline$R T$ & 14,9 & 75,0 & 25,0 & \\
\hline$R C$ & 4,3 & 50,0 & 50.0 & 0,6918 \\
\hline Distribución & & & & \\
\hline$S L$ & 8,5 & 75,0 & 25,0 & \\
\hline$L U$ & 38,3 & 66,7 & 33,3 & 0,7590 \\
\hline MU & 14,9 & 42,9 & 57,1 & 0,4029 \\
\hline MB & 38,3 & 61,1 & 38,9 & \\
\hline Territorio va & ular & & & \\
\hline$C A$ & 68,1 & 53,1 & 46,9 & 0,1109 \\
\hline$A C A$ & 36,2 & 52,9 & 47,1 & 0,3710 \\
\hline$A C M$ & 68,1 & 53,1 & 46,9 & 0,1109 \\
\hline$C P$ & 44,7 & 61,9 & 38,1 & \\
\hline AT & 29,8 & 64,3 & 35,7 & \\
\hline$A C$ & 19,1 & 55,6 & 44,4 & 0,7155 \\
\hline$A$ & 21,3 & 40,0 & 60,0 & 0,1500 \\
\hline
\end{tabular}

Abreviaturas: A, ambas (CA y CP); AC, arterias del cerebelo; ACA, arteria cerebral anterior; ACM, arteria cerebral media; AT, arterias del tronco encefálico; CA, circulación anterior; CC, cuerpo calloso; CP, circulación posterior; CR, corona radiada y centro semioval; IT, infratentorial; LU, lesión única; $\mathrm{MB}$, lesiones múltiples bilaterales; MU, lesiones múltiples unilaterales; NB, núcleos de la base; PV, periventricular; RC, región del cerebelo; RT, región del tronco encefálico; SC, fibras en U subcorticales; SG, sustancia gris cortical; SL, sin lesiones; ST, supratentorial.

El 78,7\% del total de los pacientes tuvo afectación del nivel supratentorial (ST), de los cuales el 63,2\% fue HITS-positivo y el 36,8\% HITS-negativo.

Considerando como $100 \%$ al total de pacientes con lesiones en cada localización, presentaron HITS-positivos en el DTC: el 61,5\% de los pacientes con lesiones en SG, el $60 \%$ de NB (-Fig. 6), el 83,3\% de SC (-Fig. 7), el 64,3\% de PV, el $47,8 \%$ de CR y el $50 \%$ de CC. El porcentaje restante para cada localización corresponde a los pacientes con HITS-negativos.

El 19,1\% del total de los pacientes tuvo afectación del nivel infratentorial (IT), de los cuales el 63,6\% fue HITS-positivo y el 36,4\% HITS-negativo.

Considerando como $100 \%$ al total de pacientes con lesiones en cada localización, presentaron HITS-positivos en el DTC: el 75\% del total de pacientes con lesiones en RT y el $50 \%$ de aquellos con lesiones en RC. El porcentaje restante 


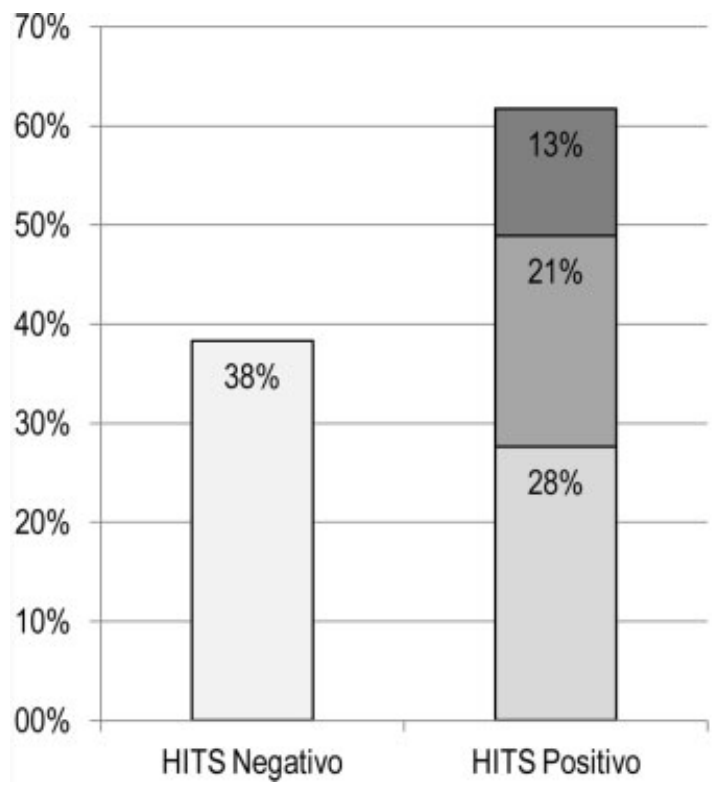

Fig. 4 Resultados de la muestra, según presencia o ausencia de HITS, y su clase. C0: Sin HITS. C1: menos de 10 HITS. C2: entre 10 y 25 HITS sin cortina. C3: HITS en cortina.

para cada localización corresponde a los pacientes con HITSnegativos.

Destacamos que se registró una relación significativa entre pacientes HITS-positivo y aquellos con lesiones a nivel SC (p 0,0294).

\section{Distribución lesional}

El 8,5\% de los pacientes se presentó SL en encéfalo, el 38,3\% con LU, el 14,9\% con lesiones MU y el 38,3\% con lesiones MB.

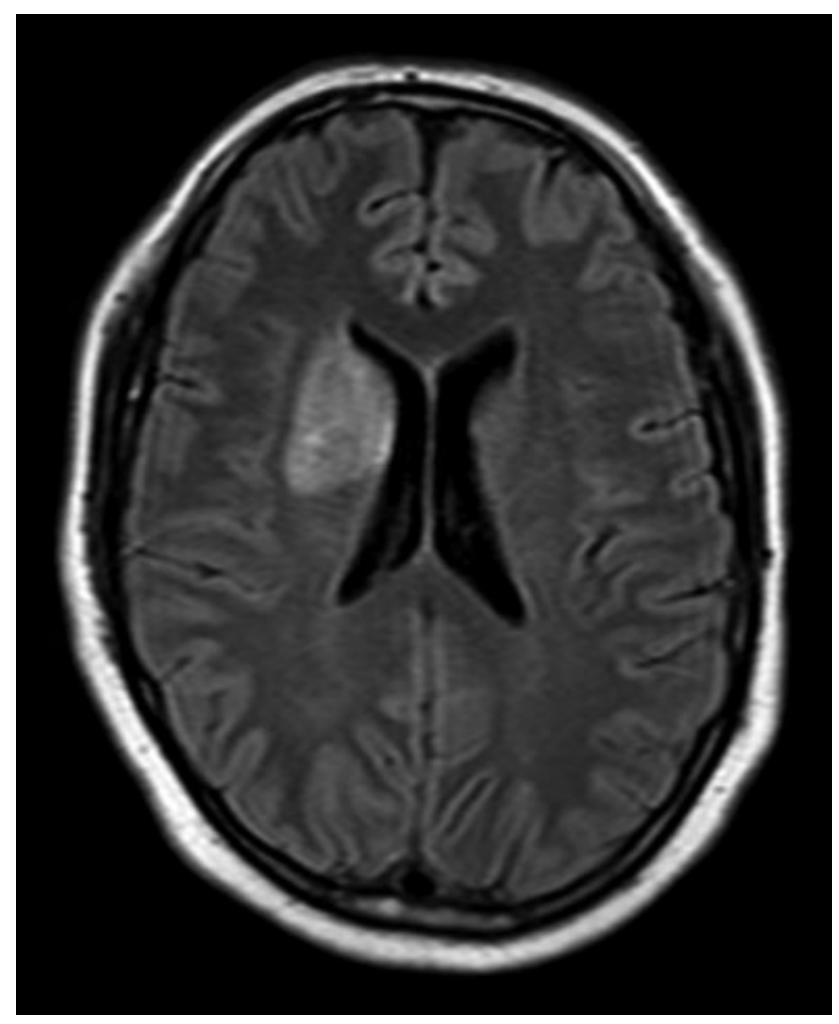

Fig. 6 Imagen en secuencia T2 FLAIR en plano axial que muestra una lesión afectando Núcleos de la Base (NB), así como la sustancia blanca de la Corona radiada y Centro semioval (CR) del hemisferio cerebral derecho.

Presentaron HITS positivos: el $75 \%$ de los pacientes SL, el $66,7 \%$ de $\mathrm{LU}$, el $42,9 \%$ de MU y 61,1\% de MB (-Tabla 2 y -Fig. 8).

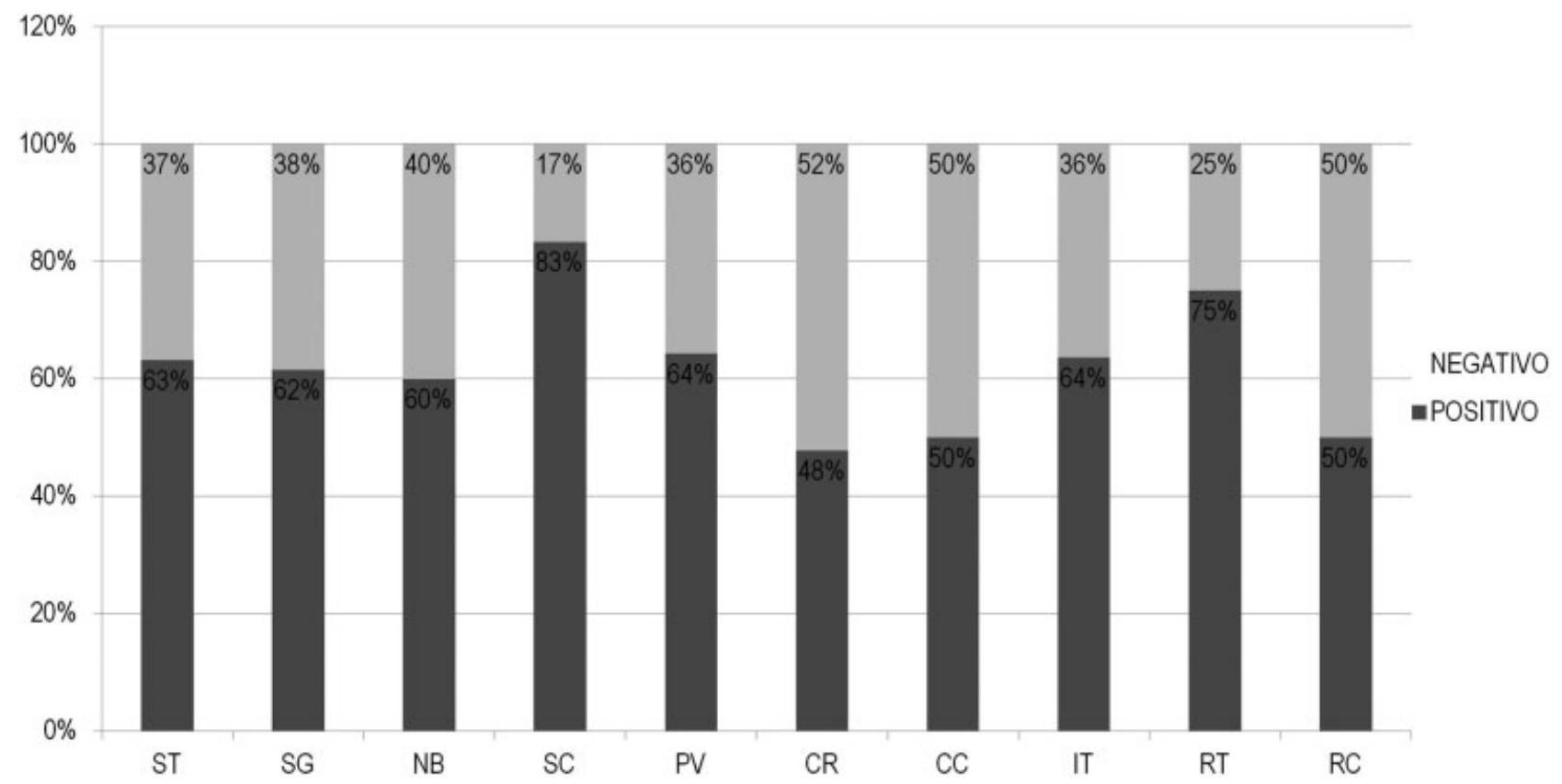

Fig. 5 Localización lesional, según resultados de HITS positivo o negativo. ST: supratentorial, SG: sustancia gris cortical, NB: núcleos de la base, SC: fibras en U subcorticales, PV: periventricular, CR: corona radiada y centro semioval, CC: cuerpo calloso, IT: infratentorial, RT: región del tronco encefálico, RC: región del cerebelo. 


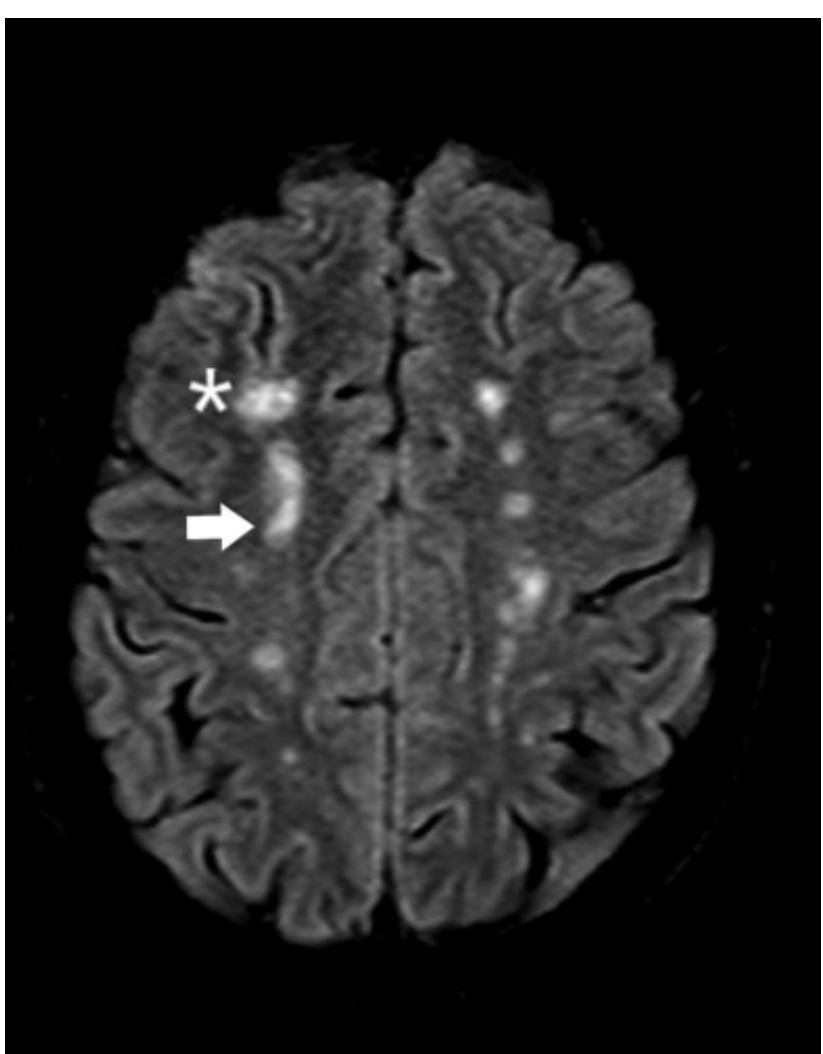

Fig. 7 Imagen T2 FLAIR en plano axial que muestra lesiones en sustancia blanca de centros semiovales (flecha blanca), con una lesión que afecta fibras en $\mathrm{U}$ subcorticales $\left(^{*}\right)$.
No se registraron asociaciones estadísticamente significativas entre la distribución lesional y la presencia de HITS. Existen dos situaciones que registran valores $p$ cercanos a los requeridos para la significancia, a saber: La asociación de HITS-positivo clase C1 con LU (p 0,0911), y los HITS-positivo C2 y las lesiones MB (p 0,0649).

\section{Territorio vascular afectado}

Teniendo en cuenta que pueden coexistir dos o más territorios vasculares afectados en un mismo paciente, observamos que (-Tabla 2 y -Fig. 9):

El 68,1\% del total de los pacientes tuvo afectación de la CA, de los cuales el 53,1\% fue HITS-positivo y el 46,9\% HITSnegativo. De los pacientes con lesiones en la CA, mostraron HITS-positivos: el 52\% de los pacientes con afectación de la ACA y el 54,29\% de la ACM.

El 44,7\% del total de los pacientes tuvo afectación de la CP, de los cuales el $61,9 \%$ fue HITS-positivo y el $38,1 \%$ HITSnegativo. De los pacientes con lesiones en la $\mathrm{CP}$, mostraron HITS-positivos: el 64,3\% de los pacientes tuvieron afectación de las AT y el 55,6\% de las AC.

Destacamos que encontramos una diferencia estadísticamente significativa entre los pacientes con HITSpositivos clase $\mathrm{C} 2 \mathrm{y}$ la presencia de lesiones localizadas a nivel de la CP $(0,0275)$. No se encontraron otras diferencias estadísticamente significativas entre el territorio vascular afectado y la presencia o ausencia de HITS.

Por otro lado, hemos observado que la localización de lesiones más frecuente en pacientes con HITS-positivos es a nivel de las fibras en U subcorticales. Se observó que la mayoría de los pacientes con HITS-positivos con lesiones SC (72\%), no presentaba FR cardiovasculares, y que sólo el 28\%

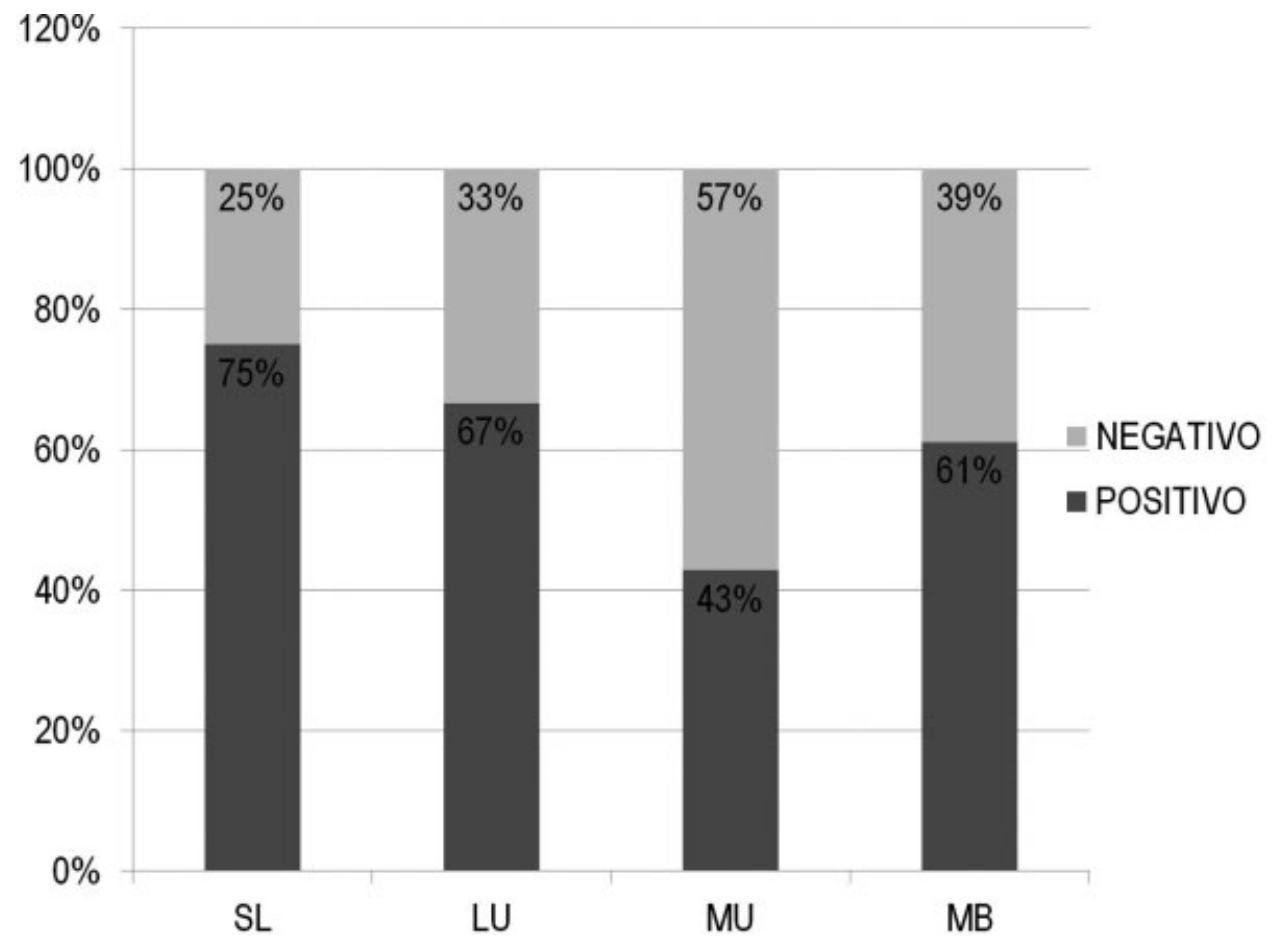

Fig. 8 Distribución lesional, según resultados de HITS positivo o negativo. SL: sin lesiones, LU: lesión única, MU: lesiones múltiples unilaterales, MB: lesiones múltiples bilaterales. 


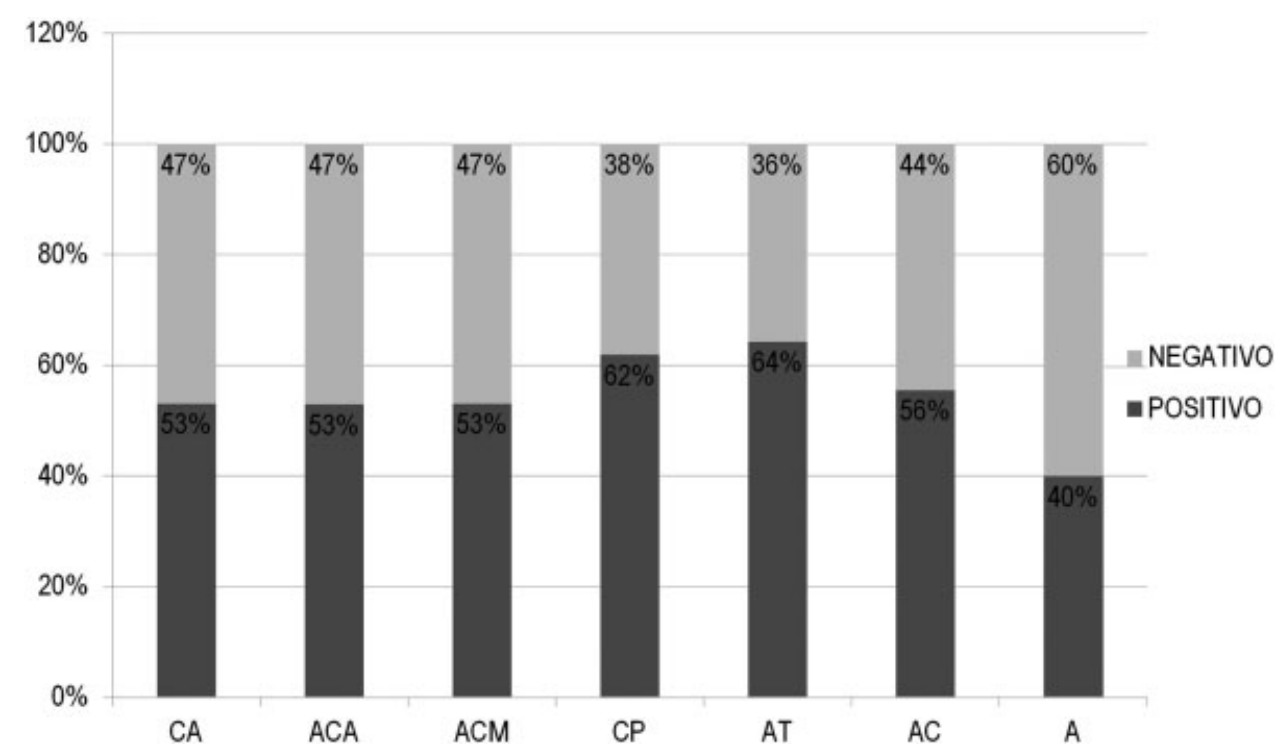

Fig. 9 Territorio vascular afectado por la lesión, según resultados de HITS positivo o negativo. CA: circulación anterior, ACA: arteria cerebral anterior, ACM: arteria cerebral media, CP: circulación posterior, AT: arterias del tronco encefálico, AC: arterias del cerebelo, A: ambas (CA y CP).

presentaba FR relevantes (p 0,2111 - no significativa). De manera similar, la mayoría de pacientes con HITS-positivos con lesiones MB (79\%) no presentaba FR y solo el 21\% presentaba FR relevantes ( $p$ 0,1985 - no significativa).

\section{Discusión}

Si bien el protocolo de estudio con RM incluyó secuencias de DWI y mapa ADC para la detección de lesiones agudas, en nuestro estudio, al igual que en el de Huang, ${ }^{25}$ se han utilizado secuencias FLAIR para el análisis de la localización de lesiones isquémicas recientes y antiguas. Las secuencias adicionales ayudaron a caracterizar las lesiones y a seleccionar aquellas que orientaban a una causa embólica.

En cuanto a la localización lesional, Boutet ${ }^{26}$ ha descripto una relación estadísticamente significativa entre pacientes con FOP y lesiones localizadas a nivel de las fibras en $U$ subcorticales. Dicha relación también fue observada en otros estudios, aunque de manera no significativa. ${ }^{25,27-30}$ Nuestros hallazgos concuerdan con los de Boutet. ${ }^{26}$ ya que el $83,3 \%$ de los pacientes con lesiones a nivel SC presenta HITS-positivos y el 16,7\% HITS-negativos (p 0,0294). También hemos observado una mayor afectación del nivel ST en pacientes HITS-positivos (63,2\%), aunque estadísticamente no significativa.

En cuanto a la distribución lesional en RM, los estudios de Huang, ${ }^{25}$ Boutet, ${ }^{26}$ Lamy, ${ }^{27}$ Liu, ${ }^{28}$ Jauss, ${ }^{29}$ Santamarina ${ }^{30}$ y Feurer, ${ }^{31}$ describen una distribución bilateral con lesiones múltiples en pacientes con FOP, hallazgo que concuerda con los resultados obtenidos en nuestro estudio (61,1\% de lesiones MB corresponden a HITS-positivos). Adicionalmente, hemos encontrado una alta proporción de pacientes con LU (66,7\% de LU corresponden a HITS-positivos). Ninguna de ellas ha mostrado ser estadísticamente significativa. Sin embargo, de esos hallazgos, se puede formular la hipótesis de que en pacientes menores de 65 años que presentan lesiones múltiples bilaterales o una lesión única hiperintensa en secuencias FLAIR con predominio subcortical, sin otra causa aparente del origen de las mismas, sugiere sospechar como etiología la posibilidad de una embolia paradójica a partir de un FOP. Mencionaremos aquí que, si bien existe una alta proporción de pacientes SL con HITS-positivos (75\%), ese valor carece de relevancia y significancia estadística, ya que la cantidad total de pacientes sin lesiones fue muy reducida (8,5\%).

En cuanto a los territorios vasculares afectados, la mayoría de los estudios antes mencionados describen una afectación ligeramente mayor de la CA en pacientes con FOP (territorios vasculares de la ACA y ACM), aunque en ninguno de ellos se ha logrado establecer una asociación estadísticamente significativa. ${ }^{25-28,31}$ Con respecto a la $\mathrm{CP}$, tres autores describen una asociación estadísticamente significativa con lesiones causadas por FOP: Boutet $^{26}$ en el territorio de la arteria cerebral posterior, Lamy $^{27}$ en el territorio de la arteria cerebelosa superior, y Jauss, ${ }^{29}$ quien considera el total del territorio de la circulación posterior. Nuestros hallazgos reflejan una predominancia no significativa de afectación de la CP $(61,9 \%)$ con respecto a la CA (53,1\%) en pacientes con HITS-positivos. A este respecto, destacamos que el subgrupo de pacientes con HITS moderados (C2), mostró una relación significativa ( $p \quad 0,0275)$ con lesiones en la $\mathrm{CP}$, lo cual concuerda con las observaciones descriptas por Jauss. ${ }^{29}$

En cuanto a la relación de pacientes con FR cardiovasculares y la presencia de HITS, podemos mencionar que, en nuestra muestra, cuando un paciente tuvo lesiones MB de localización SC, sin FR que las justificaran, hubo una mayor probabilidad de que las mismas se debieran a un FOP. Es probable que, debido al reducido tamaño de la muestra, los valores de $\mathrm{p}$ no sean estadísticamente significativos ( $p$ 0,2111 para lesiones SC y 0,1985 para lesiones MB).

De los estudios mencionados anteriormente, todos han sido realizados obteniendo imágenes a partir de resonadores magnéticos de 1.5 Tesla (T), con excepción de Huang, ${ }^{25}$ que ha utilizado uno de 3T. Ese último, es el único estudio que valoró la carga lesional utilizando la secuencia FLAIR, con respaldo de imágenes ponderadas en T2 como información 
adicional. Los otros estudios han utilizado secuencias DWI y mapas de ADC para valorar lesiones en etapas agudas, sin considerar las lesiones antiguas visibles por RM. ${ }^{26,27,29-31}$ Otro estudio ha considerado lesiones visibles sólo en imágenes ponderadas en T2, lo que, en nuestro caso, hubiera dificultado la identificación de potenciales falsos positivos, como dilataciones de espacios peri-vasculares de Virchow-Robin o bien dificultado la visualización de lesiones periventriculares o corticales. ${ }^{28}$ Nuestro protocolo de estudio incluyó, entre otras, secuencias ponderadas en T2, DWI y también FLAIR, utilizando esa última para el análisis de las lesiones. Al igual que Handke, ${ }^{32}$ no hemos encontrado una asociación entre el desarrollo de embolias paradójicas por FOP y la variable edad.

En cuanto a la relación entre los resultados del DTC y el Ecocardiograma Transesofágico (ETE), en pacientes con HITSpositivos, el $58 \%$ presentó FOP en ETE y el $42 \%$ fue negativo. En pacientes con HITS-negativos, el $100 \%$ tuvo FOP negativo en el ETE (valor p bilateral 0,00035). Si en el DTC el resultado es HITS-negativo, existe una asociación estadísticamente significativa con FOP negativo (p 0,0003). Si los HITS son positivos, la asociación con FOP es estadísticamente no significativa, por lo tanto, dicha situación debería confirmarse con ETE.

Al igual que la mayoría de los grupos de estudio mencionados, hemos encontrado la limitación de contar con un tamaño de muestra reducido, lo cual debe ser tenido en cuenta en el análisis estadístico resultante en nuestro trabajo. De la misma manera, creemos que no es posible diferenciar por medio de las imágenes de RM si las lesiones fueron a causa de embolias paradójicas o de fuentes cardioembólicas, en las cuales la literatura describe una mayor afectación de la circulación anterior. Otra limitación de nuestro estudio es la realización del DTC a cargo de un único observador. Consideramos de interés para próximos estudios evaluar la variabilidad inter-observador de la técnica.

\section{Conclusión}

En pacientes menores a 65 años con antecedentes de ACV y sospecha de FOP con HITS-positivos en DTC, en RM de encéfalo, predominan las lesiones a nivel de las fibras en $\mathrm{U}$ subcorticales. Las mismas suelen ser LU o lesiones múltiples con distribución bilateralmente simétrica. En pacientes con HITS moderados, predomina la afectación del territorio vascular de la circulación posterior.

Protección de personas y animales

Los autores declaran que para esta investigación no se han realizado experimentos en seres humanos ni en animales.

Confidencialidad de los datos

Los autores declaran que en este artículo no aparecen datos de pacientes.

Derecho a la privacidad y consentimiento informado Los autores declaran que en este artículo no aparecen datos de pacientes.
Conflicto de Intereses

Los autores declaran no tener ningún conflicto de intereses, excepto el Dr. Surur, que declara como posible conflicto de interés, ser editor responsable de la Revista Argentina de Radiología.

\section{Bibliografía}

1 Benjamin EJ, Blaha MJ, Chiuve SE, Cushman M, Das SR, Deo R, et al. Heart disease and stroke statistics-2017 update: a report from the American Heart Association. Circulation 2017;135(10): e146-e603

2 Go AS, Mozaffarian D, Roger VL, Benjamin EJ, Berry JD, Blaha MJ, et al. J. Heart disease and stroke statistics-2014 update: a report from the American Heart Association. Circulation 2014;129(03): e28-e292

3 Melcon CM, Melcon MO. Prevalence of stroke in an Argentine community. Neuroepidemiology 2006;27(02):81-88

4 Flossmann E, Schulz UG, Rothwell PM. Systematic review of methods and results of studies of the genetic epidemiology of ischemic stroke. Stroke 2004;35(01):212-227

5 Guiraud V, Amor MB, Mas JL, Touzé E. Triggers of ischemic stroke: a systematic review. Stroke 2010;41(11):2669-2677

6 Adams HP, Bendixen BH, Kappelle LJ, Biller J, Love BB, Gordon DL, et al. Classification of subtype of acute ischemic stroke. Definitions for use in a multicenter clinical trial. TOAST. Trial of Org 10172 in Acute Stroke Treatment. Stroke 1993;24(01):35-41

7 Sher K, Shah S, Kumar S. Etiologic Patterns of Ischaemic Stroke in Young Adults. J Coll Physicians Surg Pak 2013;23(07):472-475

8 Sacco RL, Ellenberg JH, Mohr JP, Tatemichi TK, Hier DB, Price TR, et al. Infarcts of undetermined cause: the NINCDS Stroke Data Bank. Ann Neurol 1989;25(04):382-390

9 Petty GW, Brown RD Jr, Whisnant JP, Sicks JD, O’Fallon WM, Wiebers DO. Ischemic stroke subtypes: a population-based study of incidence and risk factors. Stroke 1999;30(12):2513

10 Kolominsky-Rabas PL, Weber M, Gefeller O, Neundoerfer B, Heuschmann PU. Epidemiology of ischemic stroke subtypes according to TOAST criteria: incidence, recurrence, and longterm survival in ischemic stroke subtypes: a population-based study. Stroke 2001;32(12):2735-2740

11 Schulz UG, Rothwell PM. Differences in vascular risk factors between etiological subtypes of ischemic stroke: importance of population-based studies. Stroke 2003;34(08):2050-2059

12 Schneider AT, Kissela B, Woo D, Kleindorfer D, Alwell K, Miller R, et al. Ischemic stroke subtypes: a population-based study of incidence rates among blacks and whites. Stroke 2004;35(07): 1552-1556

13 Lee BI, Nam HS, Heo JH, Kim DI, Yonsei Stroke Team. Yonsei Stroke Registry. Analysis of 1,000 patients with acute cerebral infarctions. Cerebrovasc Dis 2001;12(03):145-151

14 Bang OY, Lee PH, Joo SY, Lee JS, Joo IS, Huh K. Frequency and mechanisms of stroke recurrence after cryptogenic stroke. Ann Neurol 2003;54(02):227-234

15 Bailey C, Allaqaband S, Bajwa T. Current Management of Patients with Patent Foramen Ovale and Cryptogenic Stroke: Our Experience and Review of the Literature. WMJ 2004;103(04):32-36

16 Chan M, Nadareishvili Z, Norris J. Diagnostic Strategies in Young Patients with Ischemic Stroke in Canada. Can J Neurol Sci 2000;27 (02):120-124

17 Finsterer J. Management of cryptogenic stroke. Acta Neurol Belg 2010;11(02):135-147

18 Handke M, Harloff A, Olschewski M, Hetzel A, Geibel A. Patent Foramen Ovale and Cryptogenic Stroke in Older Patients. N Engl J Med 2007;357:2262-2268

19 Overell JR, Bone I, Less KR. Interatrial septal abnormalities and stroke: a meta-analysis of case control studies. Neurology 2000; 55(08):1172-1179 
20 Steiner M, Di Tullio M, Rundek T, Gan R, Chen X, Liguori C, et al. Patent Foramen Ovale Size and Embolic Brain Imaging Findings Among Patients With Ischemic Stroke. Stroke 1998;29(05): 944-948

21 Jauch EC, Saver JL, Adams HPJr, Bruno A, Connors JJ, Demaerschalk $\mathrm{BM}$, et al. Guidelines for the early management of patients with acute ischemic stroke: a guideline for healthcare professionals from the American Heart Association/American Stroke Association. Stroke 2013;44(03):870-947

22 Blersch W, Draganski B, Holmer S, Koch H, Schlachetzki F, Bogdahn U, et al. Transcranial Duplex Sonography in the Detection of Patent Foramen Ovale. Radiology 2002;225(03):693-699

23 Teague SM, Sharma MK. Detection of paradoxical cerebral echo contrast embolization by transcranial Doppler ultrasound. Stroke $1991 ; 22(06): 740-745$

24 Basic identification criteria of Doppler microembolic signals. Consensus Committee of the Ninth Cerebral Hemodynamic Symposium. Stroke 1995;26(06):1123

25 Huang YY, Shao B, Ni XD, Li JC. Differential lesion patterns on T2weighted magnetic resonance imaging and fluid-attenuated inversion recovery sequences in cryptogenic stroke patients with patent foramen ovale. J Stroke Cerebrovasc Dis 2014;23 (06):1690-1695

26 Boutet C, Rouffiange-Leclair L, Garnier P, Quenet S, Delsart D, Varvat $\mathrm{J}$, et al. Brain magnetic resonance imaging findings in cryptogenic stroke patients under 60 years with patent foramen ovale. Eur J Radiol 2014;83(05):824-828

27 Lamy C, Giannesini C, Zuber M, Arquizan C, Meder JF, Trystram D, et al. Clinical and imaging findings in cryptogenic stroke patients with and without patent foramen ovale the PFO-ASA study. Stroke 2002;33(03):706-711

28 Liu JR, Plötz BM, Rohr A, Stingele R, Jansen O, Alfke K. Association of right-to-left shunt with frontal white matter lesions in T2weighted MR imaging of stroke patients. Neuroradiology 2009;51 (05):299-304

29 Jauss M, Wessels T, Trittmacher S, Allendörfer J, Kaps M. Embolic lesion pattern in stroke patients with patent foramen ovale compared with patients lacking an embolic source. Stroke 2006;37(08):2159-2161

30 Santamarina E, González-Alujas MT, Muñoz V, Rovira A, Rubiera M, Ribó M, et al. Stroke patients with cardiac atrial septal abnormalities: differential infarct patterns on DWI. J Neuroimaging 2006;16(04):334-340

31 Feurer R, Sadikovic S, Esposito L, Schwarze J, Bockelbrink A, Hemmer $B$, et al. Lesion patterns in patients with cryptogenic stroke with and without right-to-left-shunt. Eur J Neurol 2009;16 (10):1077-1082

32 Handke M, Harloff A, Bode C, Geibel A. Patent foramen ovale and cryptogenic stroke: a matter of age? Semin Thromb Hemost 2009; 35(05):505-514 